\title{
Pseudoaneurysm of left ventricle Report of a case diagnosed by angiography and successfully repaired
}

\author{
M. Gueron, M. Hirsch, K. Venderman, H. Freund, and J. Borman \\ From the Cardiac Laboratory and Department of Radiology, The Negev Central Hospital, Beersheva; \\ The Department of Medicine, The Shaarei Zedek Hospital, ferusalem; and the Department of Cardiac \\ Surgery, The Hadassah University Hospital, ferusalem, Israel
}

$A$ patient with rupture of the left ventricular posterior wall with the formation of a pseudoaneurysm is reported. The underlying cause of the rupture was assumed to be myocardial infarction despite absent electrocardiographic changes of myocardial necrosis and normal coronary arteriograms. The correct diagnosis was made by angiography and the aneurysm was successfully removed.

False aneurysm of the left ventricle is extremely rare and the walls, devoid of myocardium and coronary arteries, are formed by fibrous tissue. Myocardial rupture occurs in 8 per cent of cases of myocardial infarction during the first week and is only rarely compatible with life (Gould, 1960). In those rare instances of survival, adhesions between the layers of the pericardium have confined the bleeding to a limited space. The pericardial adhesions act as a protective mechanism and prevent death by cardiac tamponade.

The present case is considered worthy of publication as being the only one correctly diagnosed before operation and in which the pseudoaneurysm was successfully removed.

\section{Case report}

A 5I-year-old married woman was admitted on 14 June I97I with a history of severe chest pains of several hours' duration, radiating to the left arm and shoulder. The patient had been hypertensive for 7 years; she denied any previous history of chest pains.

The admission electrocardiogram was normal; the SGOT 74 units, LDH 1120 units, and there was a leucocytosis of 15,000 . The chest $x$-ray showed a large cardiac silhouette probably due to left ventricular enlargement.

During the first hospital days she continued to suffer from intermittent bouts of chest pain. Her temperature rose to $38^{\circ} \mathrm{C}$ and during the following 5 weeks she had almost daily spikes to approximately $38^{\circ} \mathrm{C}$. No pericardial or pleural friction rub was heard. Numerous electrocardiograms showed mild ST elevation in the left praecordial leads $\mathrm{V}_{4}$ to V6, in the limb leads and aVL, with subsequent $T$ wave inversion in these leads without abnormal $Q$ waves.

Despite the lack of definite electrocardiographic confirmation, it was felt that the patient had probably sustained a myocardial infarction and, as such, she was treated with anticoagulants. The persistent fever with daily spikes could not be explained on this basis alone; therefore, it was assumed that she was suffering from the postmyocardial infarction syndrome.

Twenty days after admission, she was suddenly seized by severe pain localized to the left shoulder radiating to the back, accompanied by cold sweats. She transiently lost consciousness and became incontinent. The blood pressure was not obtainable. The electrocardiogram showed sinus tachycardia. The sudden episode of shock was attributed to internal bleeding, the site of which was not apparent. The patient recovered and was discharged 43 days after admission.

After discharge, she developed progressive dyspnoea and signs of severe heart failure that prompted a further admission to hospital. The physical examination revealed a patient in respiratory distress. The heart was enlarged with the apical impulse palpable in the 6th intercostal space, anterior axillary line. The cardiac impulse was widespread and heaving: a double humped impulse was felt. The second sound over the second left intercostal 
space was single and accentuated; the first apical sound was preceded by a loud fourth sound. There was a third sound heard at the apex. A loud almost pansystolic (grade 3/6) harsh seagull murmur, not present before, was heard over the entire praecordium, with maximal intensity between the apex and left axilla.

The electrocardiogram showed changes related to digitalis. The chest $x$-ray showed moderate cardiac enlargement with a large mass bulging laterally to the thoracic wall and protruding from the posterior surface of the heart (Fig. r). Left ventriculography revealed a jet of contrast emerging from the posterior wall of the left ventricle through a small perforation into the large mass overlying the left ventricle posteriorly (Fig. 2). The coronary arteriograms taken in numerous projections were normal. There were no filling defects in the mass or in the left ventricle.

The patient was operated on in November 1971. The very large fibrotic mass overlying the posterolateral wall of the left ventricle was opened and its narrow neck was freed circumferentially from its attachment to the myocardium. The communication between the pseudoaneurysm and the left ventricle was obvious. The defect was situated well back in the lateral wall, $2 \times 1 \mathrm{~cm}$ in diameter, and fenestrated. The borders of the defect were fibrotic and thin, the fibrosis continuing as the thin wall of the false aneurysm. The defect was closed, and the aneurysmal sac partially excised.

The patient was re-examined 2 months after operation.
There were no signs of heart failure and no murmurs. The electrocardiogram showed changes consistent with left ventricular hypertrophy and digitalis effect.

\section{Discussion}

True left ventricular aneurysm is not rare. However, the false type has been infrequently reported and almost never recognized before death. A review of the published reports disclosed 12 cases in which the correct diagnosis was made at necropsy (Chesler et al., I969; Hurst, Fine, and Keyes, r963; Roberts and Morrow, I967; Gobel, Vidsudh-Arom, and Edwards, I97I ; Cone and Hawley, I964; Bjornsson, I964; Ersek et al., I969).

The pathogenesis of the pseudoaneurysm in our patient is not entirely clear, since every one of the I2 patients mentioned above had severe coronary disease, but our patient had normal coronary arteriogram. While it is recognized that there are pitfalls in excluding coronary disease by arteriography we took the greatest care to avoid these and the possibility of overlooking major vessel disease seems unlikely since all five major branches in our patient were visualized in numerous positions and were normal. Furthermore, it was believed that small vessel disease could not be expected to cause a localized defect in the left ventricle.
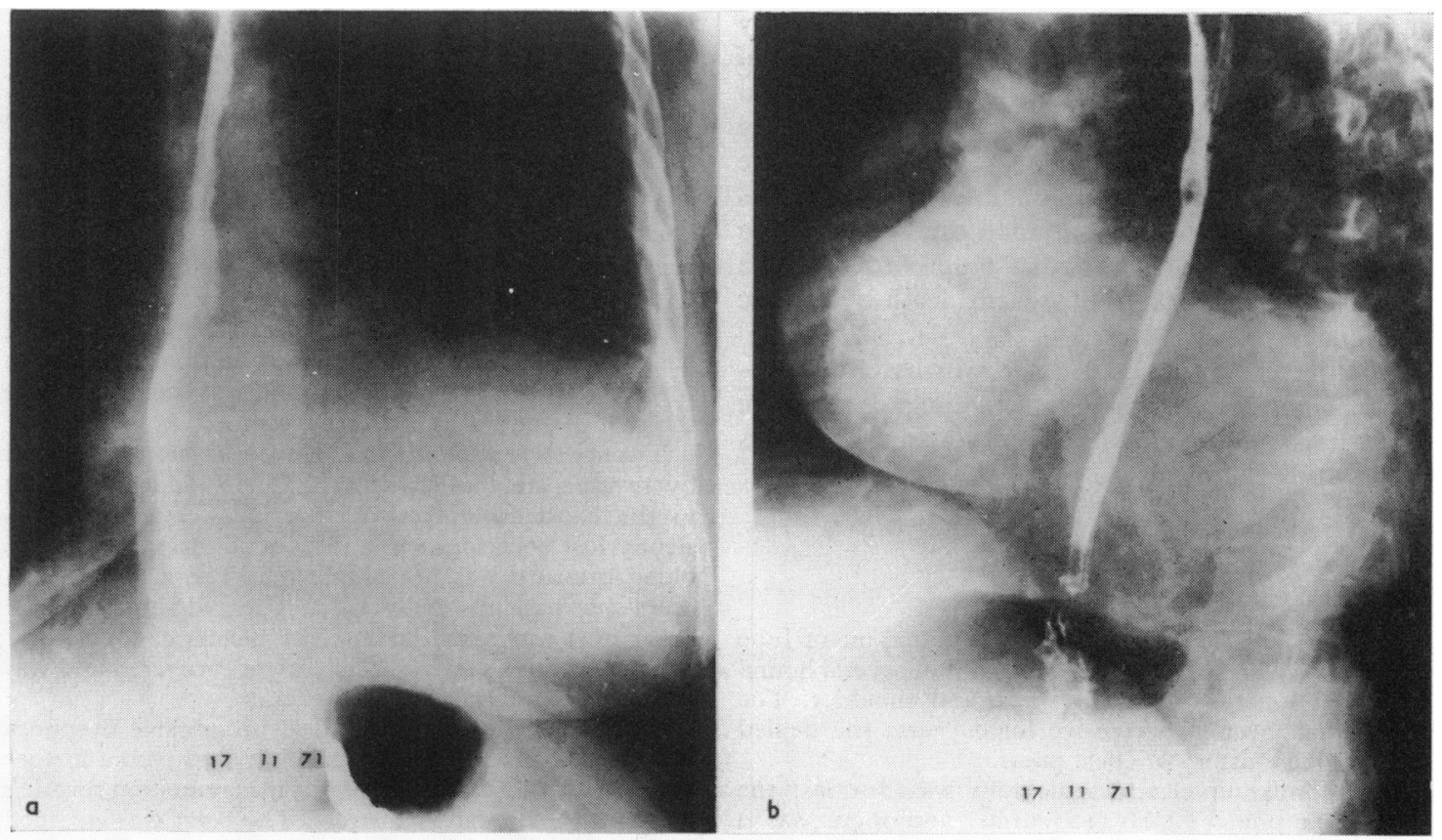

FIG. I Chest x-ray. Anteroposterior view: the left heart border is obscured by a large mass bulging laterally to the thoracic wall. Left anterior oblique view: the large mass is projecting from the posterior surface of the heart. The mass showed no pulsation. 


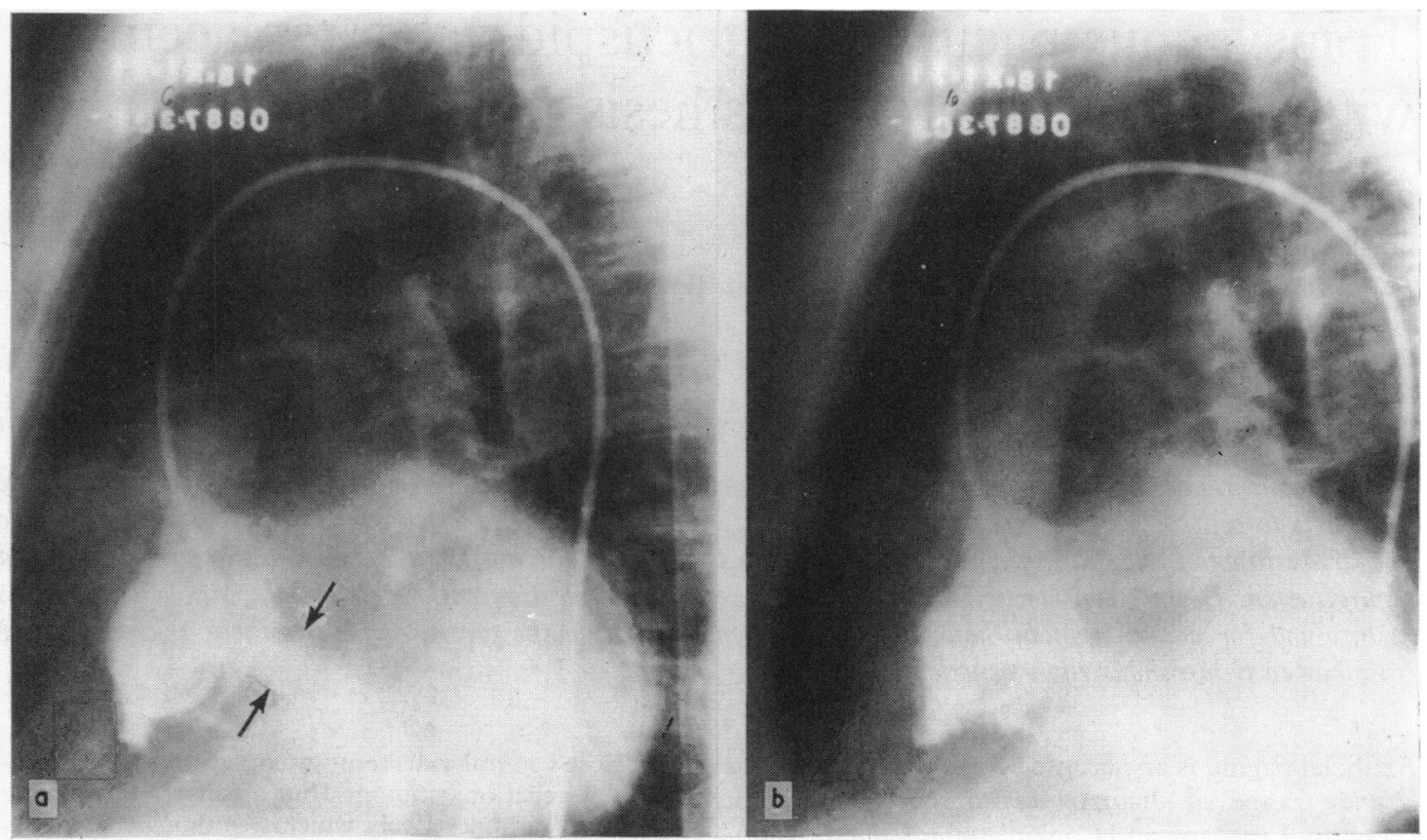

FIG. 2 Left ventriculography on lateral projection. The left ventricle is slightly displaced anteriorly by the posterior mass: a jet of contrast through a hole in the posterior wall of the left ventricle is directed into the adjacent large cavity (arrow heads).

It is suggested that the sequence of events in our patient was as follows: The clinical course, highly suggestive of myocardial infarction, was probably complicated by the postmyocardial infarction syndrome with pericarditis, with subsequent formation of pericardial adhesions. The sudden shock episode, on the 20th hospital day, was caused by perforation of the left ventricle with subsequent formation of the false aneurysm. The anticoagulants may or may not have had a contributory effect, since bleeding from organizing pericarditis after myocardial necrosis is very likely in patients receiving anticoagulants.

Operation without success was attempted in one of the previously reported 12 patients (Roberts and Morrow, 1967). He was operated on with the clinical diagnosis of true ventricular aneurysm. The successful removal of the false aneurysm in our patient suggests that correct preoperative diagnosis is crucial. This requires an active investigative approach in any patient with acute infarction in whom a rapidly developing paracardiac mass suggests ventricular aneurysm.

\section{References}

Bjornsson, L. (1964). Pseudoaneurysm of the left ventricle of the heart. American fournal of Clinical Pathology, 4I, 302.

Cone, R. B., and Hawley, R. L. (1964). Pseudoaneurysm of the heart following infarction. Archives of Pathology, 77, 80.

Chesler, E., Korns, M. E., Semba, T., and Edwards, J. E. (1969). False aneurysm of the left ventricle following myocardial infarction. American fournal of Cardiology, 23, 76.

Ersek, R. A., Chesler, E., Korns, M. E., and Edwards, J. E. (I969). Spontaneous rupture of a false left ventricular aneurysm following myocardial infarction. American Heart fournal, 77, 677 .

Gobel, F. L., Vidsudh-Arom, K., and Edwards, J. E. (197I). Pseudoaneurysm of the left ventricle leading to recurrent pericardial hemorrhage. Chest, 59, 23.

Gould, S. E. (1960). Pathology of the Heart, 2nd ed. Charles C. Thomas, Springfield, Illinois.

Hurst, C. O., Fine, G., and Keyes, J. W. (1963). Pseudoaneurysm of the heart. Circulation, $28,427$.

Roberts, W. C., and Morrow, A. G. (1967). Pseudoaneurysm of the left ventricle. American fournal of Medicine, 43, 639.

Requests for reprints to Dr. M. Gueron, Cardiopulmonary Laboratory, Negev Central Hospital, P.O.B. I5I, Beersheva, Israel. 